\title{
Highly Anisotropic Suspended Planar-Array Chips with Multidimensional Sub-Micrometric Biomolecular Patterns
}

\author{
Juan Pablo Agusil, Núria Torras, Marta Duch, Jaume Esteve, Lluïsa Pérez-García, \\ Josep Samitier, and José A. Plaza*
}

Suspended planar-array (SPA) chips embody millions of individual miniaturized arrays to work in extremely small volumes. Here, the basis of a robust methodology for the fabrication of SPA silicon chips with on-demand physical and chemical anisotropies is demonstrated. Specifically, physical traits are defined during the fabrication process with special focus on the aspect ratio, branching, faceting, and size gradient of the final chips. Additionally, the chemical attributes augment the functionality of the chips with the inclusion of complete coverage or patterns of selected biomolecules on the surface of the chips with contact printing techniques, offering an extremely high versatility, not only with the choice of the pattern shape and distribution but also in the choice of biomolecular inks to pattern. This approach increases the miniaturization of printed arrays in 3D structures by two orders of magnitude compared to those previously demonstrated. Finally, functional micrometric and sub-micrometric patterned features are demonstrated with an antibody binding assay with the recognition of the printed spots with labeled antibodies from solution. The selective addition of physical and chemical attributes on the suspended chips represents the basis for future biomedical assays performed within extremely small volumes. time-consuming. Thus, platforms to obtain cumulative data requiring fewer experiments are increasingly sought after. In bioactive sensing, ${ }^{[1]}$ analyte monitoring, ${ }^{[2]}$ therapeutics screening, ${ }^{[3]}$ and other life science applications, microarrays offer the best alternative to obtain massive amounts of information while using a single device. ${ }^{[4]}$ Initially, planar arrays (PA) have been used to accumulate and compare the acquired data with individual active features immobilized on a single substrate, where the recognition event can be tracked by the position of the sensing probe, generating a multiple-response platform. Contact, noncontact, and lithographic fabrication technologies have been traditionally used to fabricate biomolecular PA.[ ${ }^{[5]}$ Soft lithography presents a promising alternative to create biomolecular microarrays over large areas $\left(>\mathrm{cm}^{2}\right){ }^{[6]}$ where microcontact printing $(\mu \mathrm{CP})^{[7]}$ is the chosen technique to fabricate low-cost and highthroughput micropatterns by direct molecular placement. In the search for further miniaturization, polymer pen lithography $(\mathrm{PPL})^{[8]}$ was developed. This technology represents the combination of the high-resolution and serial arraying technique, dip-pen nanolithography (DPN), ${ }^{\left[{ }^{[]}\right.}$and $\mu \mathrm{CP}$. PPL uses an elastomeric stamp with an array of pyramidal features
Researchers acquire data by repeating the same experiment under identical circumstances expecting that the obtained results are consistent, a process that is material and

\author{
Prof. J. Samitier \\ Department d'Enginyeria Electrònica \\ Universitat de Barcelona \\ C/Martí i Franquès 1, Barcelona 08028, Spain \\ Prof. J. Samitier \\ Centro de Investigación Biomédica en Red en \\ Bioingeniería \\ Biomateriales y Nanomedicina (CIBER-BBN) \\ Instituto de Salud Carlos III \\ C/Monforte de Lemos 3-5, Pabellón 11, Planta 0 \\ Madrid 50018, Spain \\ ${ }^{[+]}$Present address: School of Pharmacy, The University of Nottingham, \\ University Park, Nottingham NG7 2RD, England, UK
}

Dr. L. Pérez-García, ${ }^{[+]}$

Departament de Farmacologia i Química Terapèutica

Institut de Nanociència i Nanotecnologia (IN2UB)

Universitat de Barcelon

Av. Joan XXIII s/n, Barcelona 08028, Spain

Prof. J. Samitier

Nanobioengineering Group

Institute for Bioengineering of Catalonia (IBEC)

C/Baldiri i Reixac 15-21, Barcelona 08028, Spain

DOI: 10.1002/adfm.201605912 
Physical anisotropy

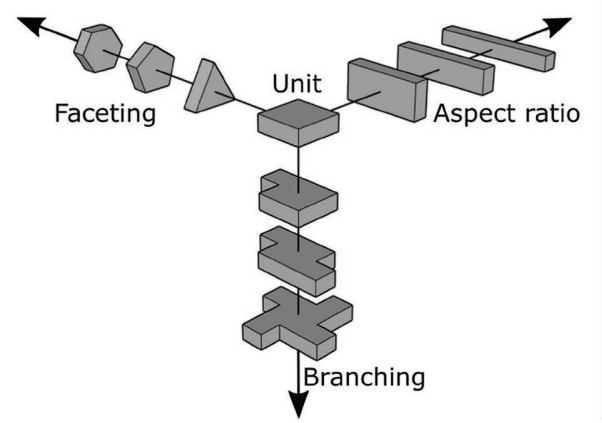

Chemical anisotropy

2D surface patterning

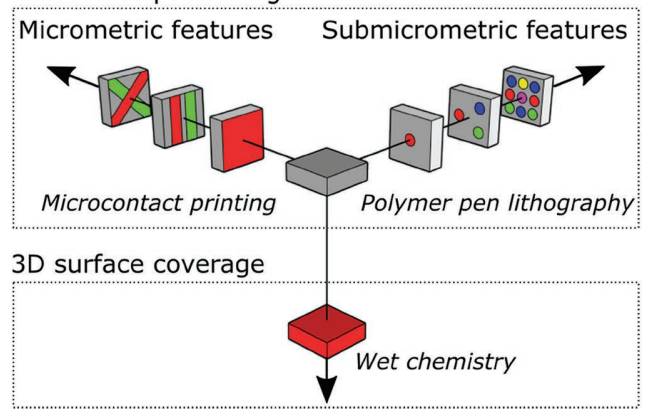

Figure 1. Anisotropic suspended planar-array (SPA) chips. The physical anisotropy is controlled during the fabrication where all shaping parameters can be tuned, allowing for a myriad of possible designs. Alternately, surface chemistry extends the functionality of the SPA chips by including reactive moieties on site, creating homogeneously coated chips or chips patterned with micro and sub-micrometric features.

to print patterns onto a substrate. The pyramids' distribution and size, along with printing force, can be tuned to obtain submicrometric patterns. ${ }^{[10,11]}$ However, traditional PPL limits the creation of complex patterns. Therefore, consecutive PPL processes with intermediate inking steps can extend the number of immobilized features deposited over the same area, promising a good alternative to create multiplexed PA. ${ }^{[6,12,13]}$

The expansion of PA into a suspended array (SA) of particles presents an alternative for faster detection within smaller samples. ${ }^{[1,14]}$ This technology relies on subpopulations of coded microparticles with unique detection probes, where the particles are free to move toward the analyte, yet the major challenge with SA is their limited applications for parallel assays due to the impossibility to identify the reaction mechanism by its spatial location in a single device, thus limiting a multidimensional probe in a single particle. Multimaterial or multiplexed particles stand as a promising alternative to obtain multiple sensing elements in a single particle. However, their complex microfabrication procedures or orthogonal chemistry functionalization limit the number of possible reactive probes on their surface. ${ }^{[15]}$

Microarray technology, with either PA or SA, has been greatly improved by the ever-increasing number of functional elements using controlled miniaturization, with major challenges on size, ${ }^{[16,17]}$ shape, and distribution of the sensing features. ${ }^{[18]}$ Therefore, in pursuit of a technology that combines the advantages of PA and SA, we developed suspended planararray (SPA) chips for sensing applications in extremely small volumes, as small as the inside of a living cell. ${ }^{19]}$ These chips consist in the dramatic miniaturization of PA, obtaining $3 \times 3 \times$ $1 \mu \mathrm{m}^{3}$ suspended $\mathrm{SiO}_{2}$ chips patterned with two $\mathrm{pH}$-dependent and one control fluorophore to sense the transmembrane transportation of protons in HeLa cells. The SPA technology uses silicon microfabrication to create micrometric $\mathrm{SiO}_{2}$ chips anchored on a substrate, which are then patterned with various inks and finally released.

As a significant step beyond, we present in this work a highly versatile approach to extend the generation of SPA chips with increased physical anisotropy, coupled with the discrete and independent biomolecular microarrays of increased complexity, with biochemical recognition capabilities for future biomedical applications. The conceptual facets of this approach are summarized in Figure 1. The chips were microfabricated following the photolithographic and etching processes to fabricate the SPA chips, where the extended physical anisotropy is obtained by the simple alternation of photomasks to obtain the desired chip geometry and shape attributes. The combination with improved patterning techniques transported the SPA chips onto the multidimensional realm with the creation of multiplexed biomolecular patterns with features ranging from the micrometric to the sub-micrometric scale, each with a unique biochemical signature. A printing setup was used to fabricate the biomolecular patterns by design on top of the anchored chips dictated by nanocombinatorics, ${ }^{[20]}$ that is, controlling precisely the physical micropattern anisotropy along the pattern distribution.

\section{Results and Discussion}

\subsection{Fabrication of Silicon Chips}

We used silicon microtechnology to fabricate the substrate, consisting of $\mathrm{SiO}_{2}$ chips fixed to the silicon wafer substrate with a subjacent anchor, following a protocol presented previously. ${ }^{[19]}$ As shown in Figure 2a, the initial 1- $\mu$ m-thick $\mathrm{SiO}_{2}$ layer was grown by wet oxidation of a silicon wafer. A subsequent standard photolithographic step using a positive photoresist defined the geometry and distribution of the chips. After the photoresist was developed, the exposed $\mathrm{SiO}_{2}$ was dry-etched. The final step nanomachined the underlying silicon using quasi-isotropic etching and later the remaining photoresist was stripped off.

\subsubsection{Physical Anisotropy in Chip Design}

The physical attributes for each anchored chip are dictated by the features designed in the photomask. Hence, the physical anisotropies can be tuned to obtain chips with specialized morphology-directed function or even allowing their assembly into more complex structures. ${ }^{[21]}$ Taking advantage of the inherent 2D photolithographic process, the lateral dimensions, shape, and distribution of the chips on the wafer can be finely adjusted. 
a
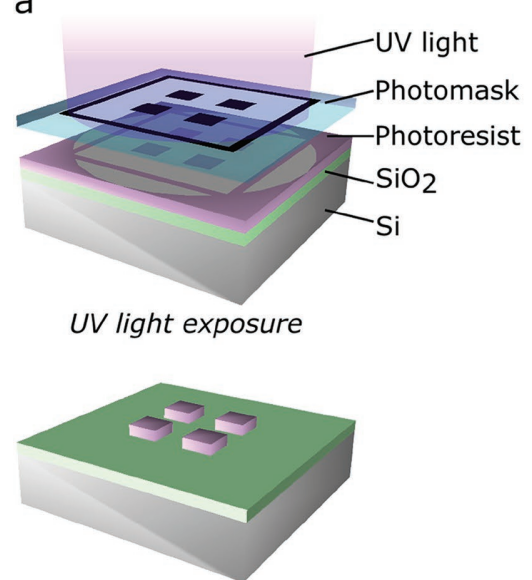

Photoresist developing
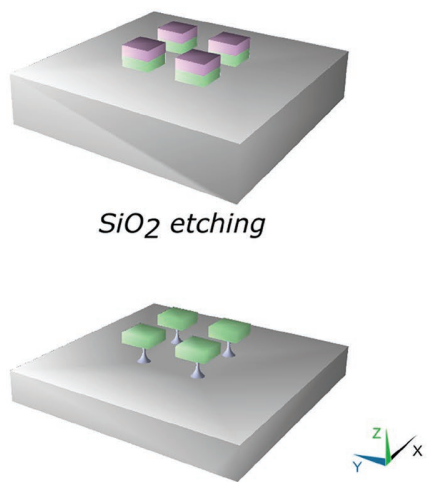

Si anisotropic etching b

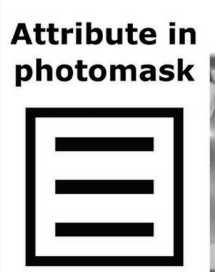

Aspect ratio
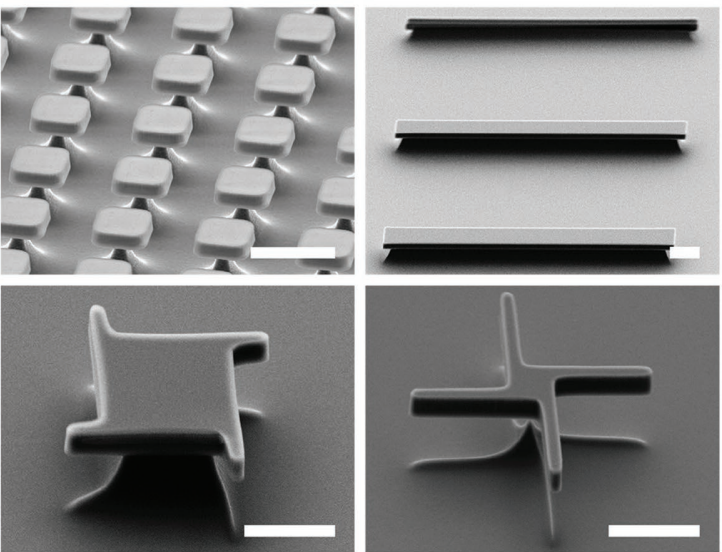

Branching
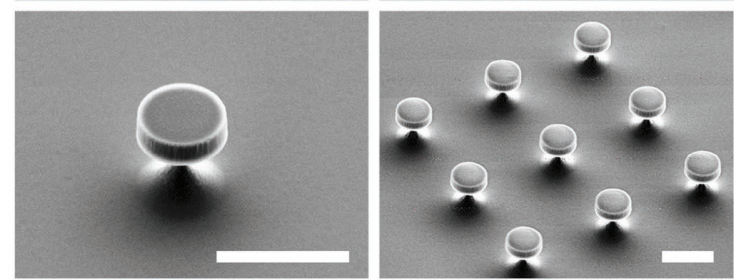

Faceting
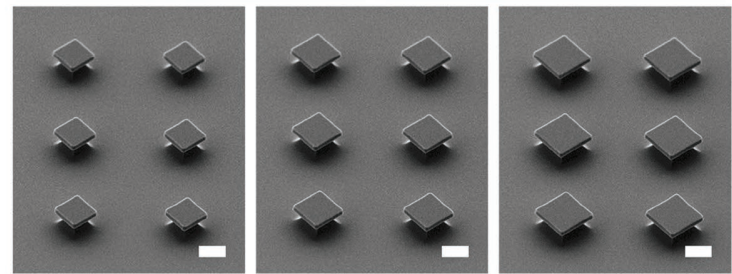

Figure 2. Versatility on the physical attributes on the chips. a) A $1 \mu \mathrm{m}$ thermally grown $\mathrm{SiO}_{2}$ layer is coated with positive photoresist and subsequently exposed to UV light through a photomask. After developing the photoresist, the exposed $\mathrm{SiO}_{2}$ was selectively etched to form the chips. A controlled final anisotropic etching nanomachined the silicon anchor beneath every $\mathrm{SiO}_{2}$ chip. b) The physical anisotropy is defined by the photomasks. The SEM images present the physical attributes in the fabricated chips, including variations in aspect ratio, branching, faceting, and size gradient. Scale bars $=5 \mu \mathrm{m}$

These parameters are defined taking into account the technical limitations of standard photolithography and hence focus on designs with features above the $500 \mathrm{~nm}$ threshold, thus avoiding unresolved elements. Additionally, it must be considered the anchor vertical-to-lateral etch ratio of $\approx 1.3: 1$ to prevent overetching of the anchor. Consequently, following this approach, the number of potential designs produced in batch is unprecedented. Figure $2 \mathrm{~b}$ presents the varied shapes obtained with the SPA technology, focusing on aspect ratio from 1:1 to 25:1 in the $x$ and $y$ planar directions, along with chips with orthogonal branching at various extensions, also, alternating chip faceting, and finally, varying size gradient.

Every physical attribute is compatible with the fabrication process, obtaining mechanically fixed chips with an integrated silicon anchor. This anchor prevents the disruption of the chips from the wafer, while its mechanical stiffness allows a future contact-printing patterning and, if required, a controlled release of the chips, by applying a later force to break the anchor.

Thus, to prevent any damage to the chips or the pattern during the controlled breakage, we used a peel-off method using an aqueous mounting medium that we previously developed. ${ }^{[19]}$
This method starts with a drop of the mounting medium placed on top of the chips and is left to solidify. The medium engulfed the chips within its matrix, and after solidification, a flexible, water-soluble membrane was obtained. This membrane can be easily manipulated to break the silicon anchors with a lateral manual force. The suspended $\mathrm{SiO}_{2}$ chips with defined physical attributes are presented in Figure 3, showing chips with b) different branching; c) chips with alternating size gradient with the dimensions ranging from $2.5 \times 2.5,4 \times 4$, up to $5 \times 5 \mu^{2}$, respectively; and d) homogeneous $3 \times 3 \mu \mathrm{m}^{2}$ chips. The images were obtained after the solidified mounting media was peeledoff and dissolved in Milli-Q water. It is clearly seen that the suspended chips maintain their physical integrity after releasing, promising an alternative for future uses with shape-dependent applications.

\subsection{Chemical Anisotropy with Surface Modification}

The resulting chips presented a constricted and independent area where a pattern could be subsequently printed. Therefore, 


\section{w.afm-journalde}
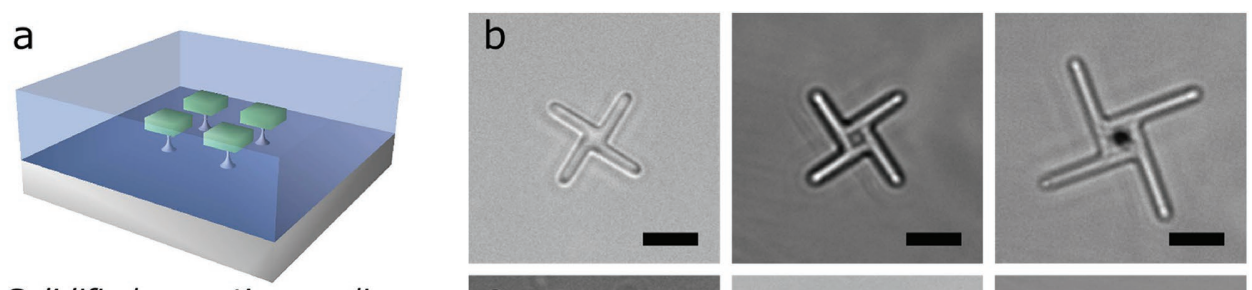

Solidified mounting medium
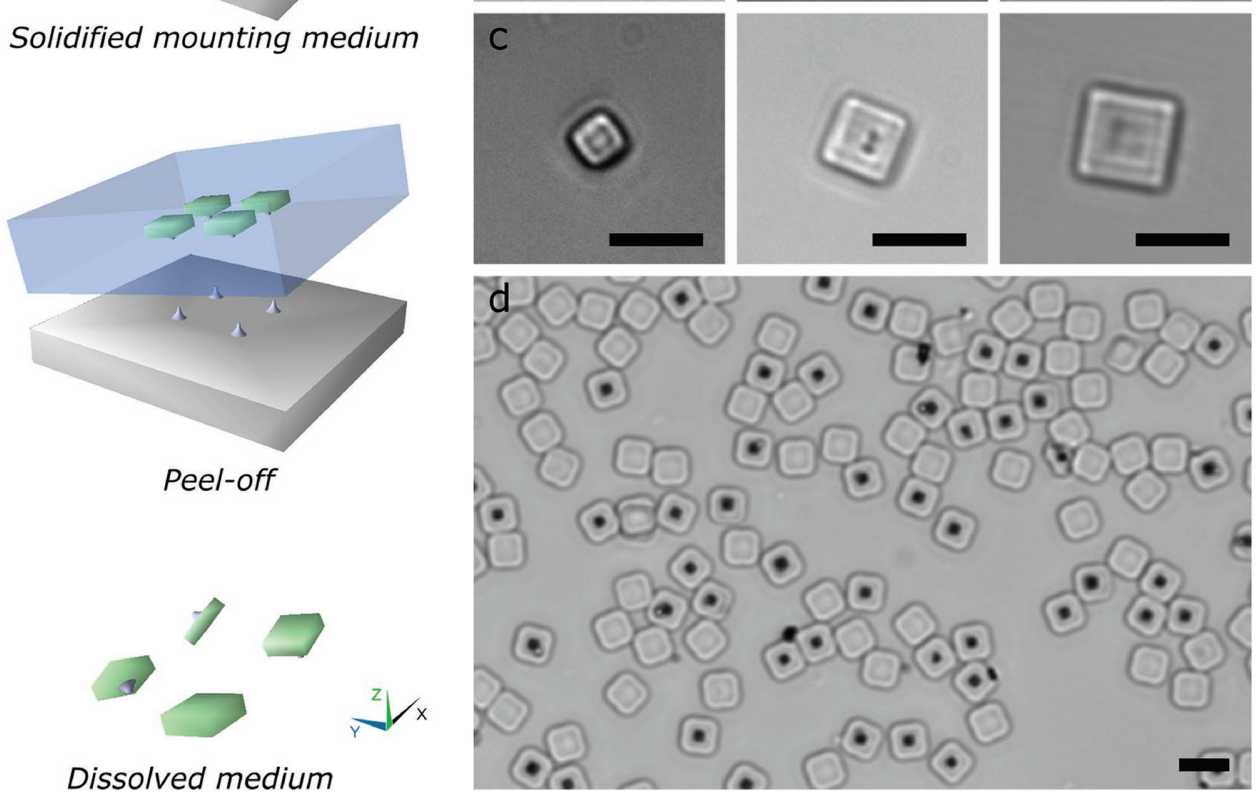

\section{Dissolved medium}

Figure 3. Released anchored microparticles. a) Lateral forces using the solidified mounting medium are applied to break the silicon anchor from every type of anchored chips. Optical microscopy images of b) branched, c) size gradient $\left(2.5 \times 2.5,4 \times 4\right.$, and $5 \times 5 \mu \mathrm{m}^{2}$, respectively), and d) homogeneous $\left(3 \times 3 \mu \mathrm{m}^{2}\right) \mathrm{SiO}_{2}$ chips. Scale bars $=5 \mu \mathrm{m}$.

we used contact-printing techniques based on polydimethylsiloxane (PDMS) stamps to pattern the surface of the chips, as these stamping techniques provide the basis for a myriad of versatile patterns on each anchored chip. The various chip shapes, when coupled with alternating printed biomolecules, can generate an outstanding number of complex combinations. To demonstrate the patterning capabilities, chips with an aspect ratio of 1:1 were fabricated following the photolithographic and etching protocols, obtaining a density of $2.7 \times 10^{6}$ chips $\mathrm{cm}^{-2}$. The chip surface was initially functionalized with an epoxysilane to generate reactive moieties readily accessible for surface chemistry along the substrate following the steps represented in Figure 4a. The complete surface modification for all different patterning approaches can be seen in Figure S1 (Supporting Information). All the chips presented the same geometric features seen in Figure $4 \mathrm{~b}$. Subsequently, our custom-made printing device with sub-micrometric translation capabilities was used to print the patterns on top of the anchored chips (Figure S2, Supporting Information). The prototype consists of a five-axis holder to fix and situate the substrate accurately under the stamp. A piezo-actuated stamp holder translates the stamp toward the printing area and includes four strain gauges to measure the printing force applied between stamp and substrate. Finally, a dedicated optical monitoring system consisting of a $10 \times$ objective, zoom lenses, and a charge coupled device (CCD) camera located on top of the stamp-substrate pair, is used to oversee the printing procedure.

\subsection{Complete 3D Surface Modification with Wet Chemistry}

An initial approach to create functionalized chips along all their surfaces was the immobilization of the molecular inks by incubation. This approach required the surface activation of all the exposed surfaces with the epoxy linker, obtained by submerging the plasma-activated substrate into an ethanolic epoxy-silane solution. After curing, the substrate could be incubated with any amine-bearing molecular ink which generated a covalently bonded monolayer with the epoxy linker molecule. This modification created a fast, single-ink, 3D modification of the anchored $\mathrm{SiO}_{2}$ chip surfaces. The concept is shown with a complete substrate incubated with Oregon Green 488 Wheat germ agglutinin (WGA) in Figure 4c.

\subsection{2. $2 D$ Surface Patterning with $\mu C P$}

In search for the introduction of multiple immobilized probes with different biochemical attributes, we next multiplied the number of printed features in a single, shared microarray. DNA, proteins, and antibodies were independently patterned on the anchored chips to create parallel testing areas on a single substrate. Previous approaches have been developed to pattern 3D anchored structures with a diameter of $40 \mu \mathrm{m}$ and pitch of $200 \mu \mathrm{m}$ with soft lithography, ${ }^{[22]}$ requiring the inked features to match the distribution of the anchored structures. Our work 


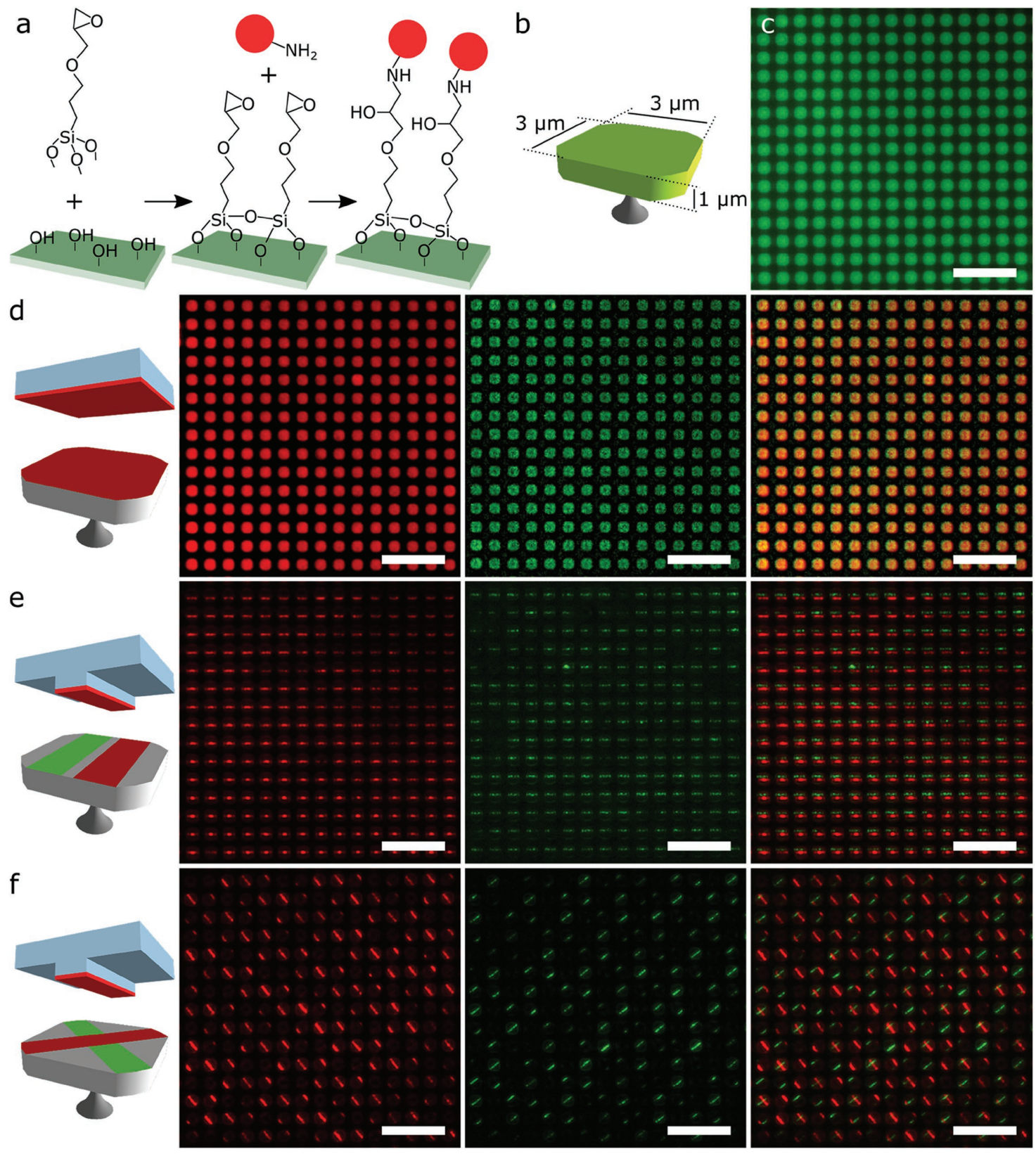

Figure 4. Immobilization of biomolecules on the chips via wet chemistry and $\mu \mathrm{CP}$. a) Functionalization strategy to endow reactive moieties to the $\mathrm{SiO}_{2}$ surface. b) Description of the dimensions of an anchored chip with the silane functionalization. c) Fluorescence microscopy images of the substrate modification with wet chemistry; the coverage is around all the exposed surface areas as represented in (b). d) The scheme represents the flat stamp used to pattern the FP- $\mathrm{NH}_{2}-\mathrm{T}$ strand (left) over the functionalized chips, and subsequently hybridized with the 5OG-3 strand (middle). The distribution of hybridized strands matches that of the printed features (composite, right). e) Representation and fluorescence microscopy images of horizontal parallel lines of Texas Red WGA (left), and Oregon Green 488 WGA (middle) patterned consecutively over a same substrate, and the overlay (right). f) Representation and fluorescence microscopy images of horizontal Texas Red WGA lines (left) patterned at a $-45^{\circ}$ angle from the substrate, along Oregon Green $488 \mathrm{WGA}$ lines (middle) at $45^{\circ}$ angle from the substrate patterned consecutively forming $90^{\circ}$ crosses, seen in the overlay (right). Scale bars $=20 \mu \mathrm{m}$.

requires tackling the same feat using a substrate two orders of magnitude smaller and with multiplexed designs. Thus, the first approach to functionalize the surface of the anchored chips was using $\mu \mathrm{CP}$ PDMS stamps, inked with fluorescent biomolecules to characterize the printing mechanism and the formation of the biomolecular monolayers. Every $\mu \mathrm{CP}$ stamp was fixed onto a glass slide inked with the biomolecule solution, and fitted into the stamp holder of the printing device. The printing force was monitored throughout the process to prevent the breakage of the silicon anchors during contact. In Figure 4d, the FP- $\mathrm{NH}_{2}-\mathrm{T}$ DNA strand modified with an amine moiety was printed with a flat PDMS stamp on top of the epoxy-functionalized substrate using direct DNA $\mu \mathrm{CP} .^{[23]}$ After leaving the epoxy-amine reaction to proceed, the substrate was washed, and the immobilized 
strand was later incubated with the complementary 5OG-3 strand labeled with Oregon Green 488 hybridizing selectively on the printed areas. This printing approach using a flat PDMS stamp allowed the creation of dense DNA arrays with a single printing process over only a single surface of the chip. The intense fluorescent signal is accounted by an efficient hybridization due to an increased access from the targets to the highly organized printed probes. ${ }^{[23]}$

To demonstrate the versatility of the printing device, we interchanged the printing patterns using different PDMS stamps, providing the means to create diverse designs on top of the anchored chips. Using a PDMS stamp consisting of $1 \mu \mathrm{m}$ parallel lines with a period of $5 \mu \mathrm{m}$, the anchored chips were patterned with two fluorescently labeled proteins to create protein lines. The substrate was positioned with a perfect alignment, following the protruding features of the PDMS stamp, obtaining the patterns shown in Figure 4e,f. The fluorescent microscopy images in Figure 4e show the parallel distribution of Texas Red WGA (red) and Oregon Green 488 WGA (green) lines along the sample. The precise control during the printing process allowed us to deposit inks with a separation of less than $1 \mu \mathrm{m}$. Consequently, by modifying the relative position of the substrate against the stamp, different patterns were generated, extending the multiplexing capabilities. The different lines obtained in Figure $4 \mathrm{f}$ extended the patterning to another dimension by alternating feature length and distribution. These lines were produced printing Texas Red WGA (red) at a $-45^{\circ}$ rotation angle between the stamp and substrate. When adding a second set of lines using Oregon Green 488 WGA (green) at $45^{\circ}$, the multiplexed complexity is dramatically increased. The composite image in the far right of Figure $4 \mathrm{f}$ demonstrates the size and distribution of the previous printed features within the same patterned substrate, first by allocating different amounts of proteins along the surface of the anchored chip, and second by generating crossed protein patterns.

\subsection{3. $2 D$ Surface Patterning with PPL}

In order to extend the possible applications of our technology, we had to create more complex micropatterns on top of each anchored chip, hence it was necessary the use of stamps with features smaller than $1 \mu \mathrm{m}$ that matched the distribution of the anchored chips. PPL was found to be the best alternative to create the required sub-micrometric patterns on top of the anchored chips.

The biomolecular subpatterns ${ }^{[6]}$ were generated with the contact printing of different inks adsorbed on the PPL stamp over the anchored chips. In an initial approach to pattern the substrate, a divided area on the stamp was coated with the first ink. This area was carefully located on top of the anchored chips, and the polymeric features transferred the ink where they contacted the chips. A second divided region of the stamp was coated with a second ink and moved to the printing area. Consecutive printing steps were followed to obtain up to five different printed features on each anchored chip. Alternatively, various individual stamps coated independently with an ink were also sequentially used to pattern the substrate. This inking method allows the creation of multiplexed patterns without the need to create complex inking protocols to coat independent printing features. ${ }^{[12]}$ The topography of the PPL stamp is presented in Figure 5a along with its profile, which agrees with the height of the theoretical potassium hydroxide $(\mathrm{KOH})$ anisotropic etching on the $\langle 111\rangle$ plane of each $3 \mu \mathrm{m}$ base pyramid $(\sqrt{2} \cdot \mathrm{base} / 2=2.12 \mu \mathrm{m})$ on the silicon wafer used as the replica mold. ${ }^{[19,24]}$ Despite the high versatility to generate sub-micropatterns with complex designs for promising biomedical applications, the PPL technology generates arbitrary patterns on the substrate, ${ }^{[25,26]}$ rendering it inappropriate for applications where the alignment between stamp and substrate is essential. Ultimately, to obtain an accurate pattern on every anchored chip, the alignment between stamp and substrate in
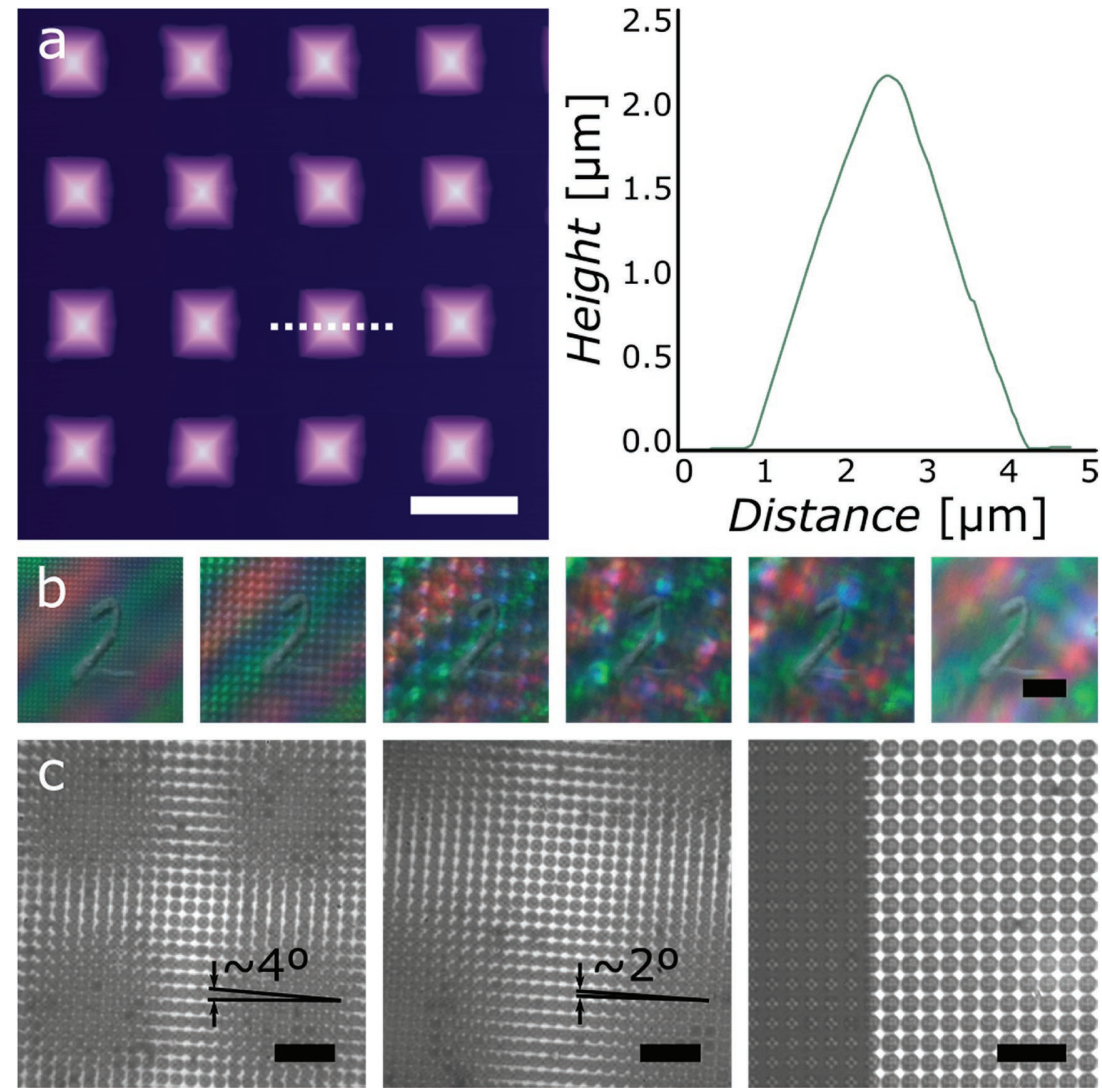

Figure 5. Characterization of the PPL stamp and angular alignment. a) Left: AFM topographical image of the PDMS PPL stamp. Scale bar $=5 \mu \mathrm{m}$. Right: Profile obtained from the dashed line on the AFM image. b) Digital photographs of a PPL stamp over the substrate at different rotation alignment. Moiré patterns are produced when the stamp and the substrate are misaligned. Scale bar $=250 \mu \mathrm{m}$. c) Optical microscopy images with the stamp and substrate with an $\approx 4^{\circ}$ and $\approx 2^{\circ}$ misalignments on the left and center, respectively, and a perfect alignment on the right. The dark vertical strip on the rightmost image shows an area outside the substrate. Scale bars $=20 \mu \mathrm{m}$. 
all 5D was imperative. Therefore, the lateral displacement of the substrate on both the $x$ and $y$ planar axes was controlled with dedicated microsteppers together with two automatized microgoniometers and a rotating stage. Moiré interference patterns were observed when the stamp and substrate were misaligned yet positioned on the same focal plane. This effect was utilized to align the substrate and stamp, similar to previous works where these patterns were used to align a substrate with nanopillars with a PDMS stamp with printing features larger than the top surface of the nanopillars. ${ }^{[27]}$ Therefore, the accurate placement of the stamp over the chips does not rely on the individual shape of the chip, but on the overall position of all the chips along the wafer. The sequential captured images of a PPL stamp on top of the substrate at different planar angles from a large angular difference to a perfect alignment are presented in Figure 5b, along with the microscopy images presenting the same effect at the microscale in Figure $5 \mathrm{c}$. A perfect alignment was achieved in all 5D when the overlaid dark interference spots disappeared from the field of view.

It is evident that each polymer pen deforms on contact with the substrate during the printing process. Hence, the exerted forces are proportional to the apex deformation, and consequently the printing protocol can be tuned to obtain spots of different sizes. ${ }^{[28]}$ Previous works have focused on the effect of patterning with a tilted stamp, where the printing contact exerted inhomogeneous pressure over the flat substrate, thus obtaining an array of features with different edge lengths. ${ }^{[20,25,29]}$ On the contrary, the five-axis substrate holder allowed the precise alignment between substrate and stamp to minimize any artefact derived from the difference in printing forces.

The printing resolution was compared with the simulated deformation of the pen under diverse pressures using the finite element method (FEM). The displacement of the polymer pen at the contact point is shown in Figure 6a. The mechanical deformation is concentrated at the apex of each pyramid, providing an increasing feature edge length as the contact progresses. PDMS has been traditionally reported to have a Young's modulus between 0.34 and $0.75 \mathrm{MPa},{ }^{[28,30]}$ hence the deformation, and consequently the printed features depend on the stiffness (form and mechanical properties of the tip) of the stamp. The simulations in Figure $6 \mathrm{~b}$ represent the deformation of the tip when $1.0 \mu \mathrm{N}$ is exerted on a polymer pen with both maximum and minimum reported moduli. As expected, the contact area increases when using a more yielding modulus. To adjust the printed spots with the simulations, a Texas Red WGA ink was pipetted onto the stamp, then loaded into the printing device, and printed at various printing forces. The images on top of Figure $6 c$ present the deformation of the polymer pen during the printing process when contacting a rigid substrate. The full width at half maximum (FWHM) of the fitted curves was used to measure the contact area of the printing pens under visible light (Figure S3, Supporting Information). Their printing signatures were characterized using the printed spots with fluorescence microscopy, obtaining the patterns shown on the bottom of Figure 6c. The lengths of the edges of both, the deformed tips and the printed features, are plotted against the printing force per pen in Figure $6 \mathrm{~d}$, along with the FEM-simulated maximum and minimum features that could be obtained with the reported Young's moduli.
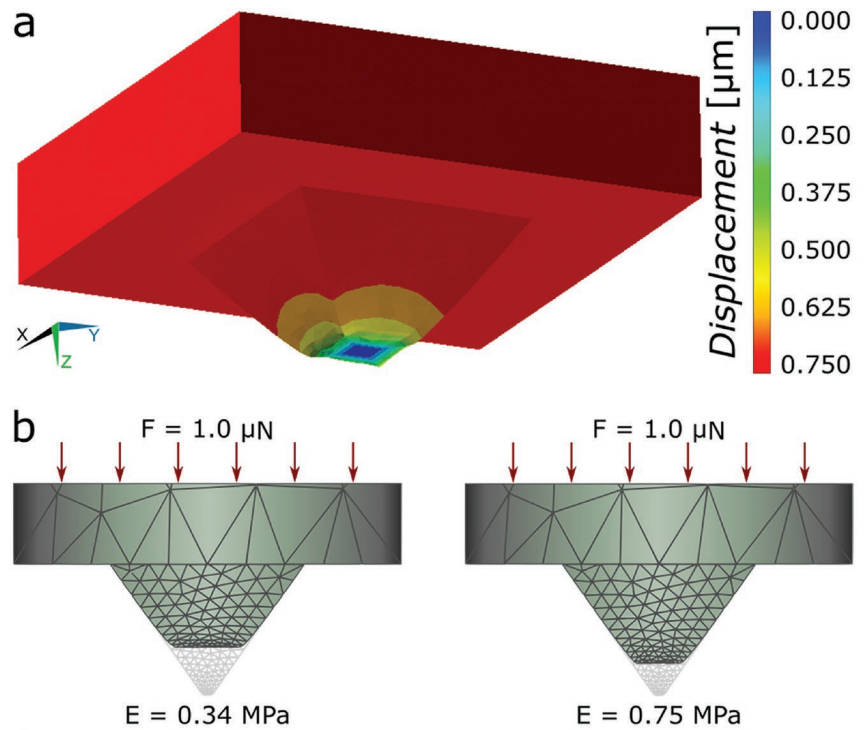

C
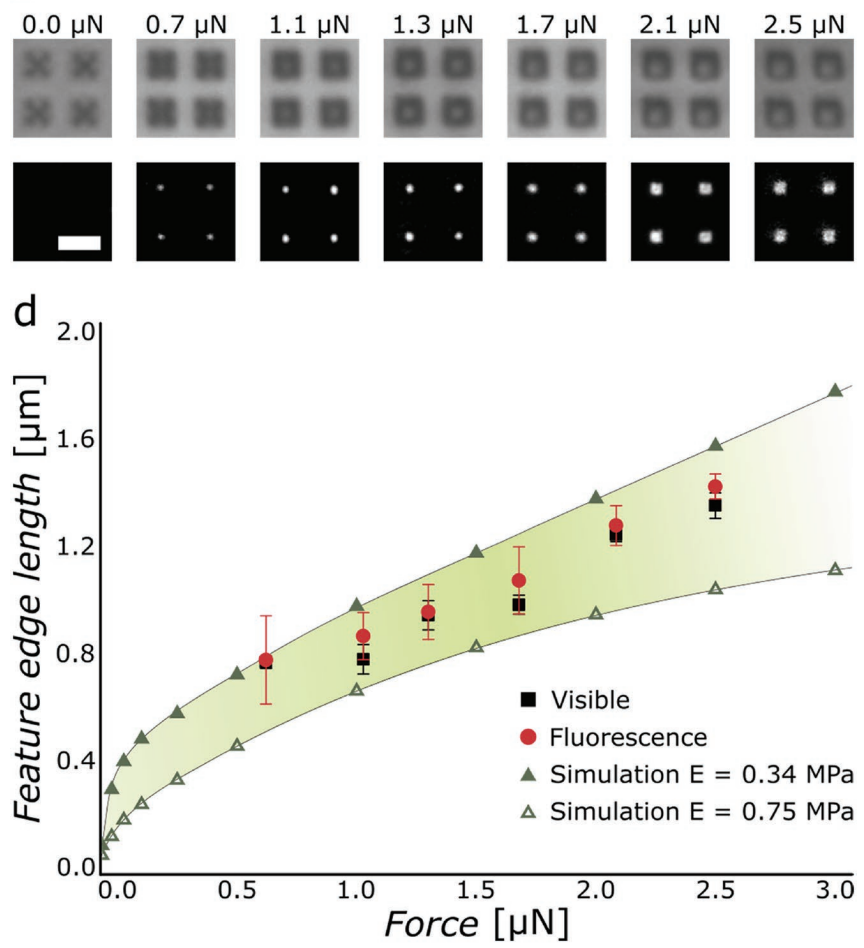

Figure 6. PPL stamp behavior under different printing forces. a) FEM of the mechanical deformation on the PDMS PPL stamp versus applied force. The displacement is given by the printing force and subsequent collapse of the tip of the elastomeric pyramid. b) Lateral view of a PDMS pen with an exerted force of $1.0 \mu \mathrm{N}$ with the reported maximum and minimum moduli. ${ }^{[28]} \mathrm{c}$ ) Top: Optical microscopy images of the PPL stamp at different printing forces in contact with a Si substrate. The force value is equivalent to the force exerted by a single PDMS pen. Bottom: Fluorescence microscopy images of patterned WGA Texas Red over a Si substrate at various printing forces. Scale bar $=5 \mu \mathrm{m}$ for both top and bottom images. d) Comparison of the simulated and experimental feature edge length of the deformed tips in contact with the substrate (visible) and of the patterned features at different printing forces (fluorescence, $n=25$ for each point). The delimiting region (green) represents the simulated feature edge length with $E=0.34 \mathrm{MPa}$ (top line) and $E=0.75 \mathrm{MPa}$ (bottom line). 
Previous reports suggest that each polymer pen experiences a change on its Young's modulus during the printing process, establishing a two-regime compression behavior. ${ }^{[10]}$ Contrarily, our simulations confirmed the gradual change on the stiffness of the pen, yet maintaining the same Young's modulus, which is due to the deformation of the rounded apex and the overall geometry of the tips. This behavior may be attributed to a rapid initial compression of the rounded apex of each pyramid with a subsequent linear behavior once the apex has collapsed. The printed patterns were collectively analyzed and subsequently compared with the FEM simulations, obtaining a calculated Young's modulus of $0.44 \mathrm{MPa}$ from our PPL stamps, fitting previously reported values. ${ }^{[28,30]}$

In our first printing approaches, a PPL PDMS stamp with no fixed solid backbone was used to pattern the anchored chips. As previously documented, the PDMS shrinkage changed the distribution of the features along the stamp. ${ }^{[31,32]}$ Using this stamp, the displacement of the printing features against the anchored chips created contact areas where the ink was successfully patterned. In a single-printing step, the misalignment generated areas without pattern, coupled with patterned features not homogeneously distributed along neighboring anchored chip (Figure S4, Supporting Information). Although not sought after, taking advantage of this phenomenon, we used multiple printing steps to create complex patterns on top of the anchored chips by translating the substrate to alternate the contact area. The steps to print up to four spots per chip are graphically demonstrated in Figure S5 (Supporting Information) with $3 \mu \mathrm{m}$ translations on both the $x$ and $y$ planar axes used to pattern the complete substrate. The fluorescent emission of Texas Red WGA patterned alternating the contact area after a $4 \times$ printing process is shown in Figure S6a (Supporting Information). Following this technical approach, a two-ink system was elaborated by introducing a secondary ink with a different fluorescence emission. After the initial Texas Red WGA pattern, the substrate was relocated under the Oregon Green 488 WGA-inked area of the shrunken stamp and the same printing protocol was followed. The two-color nonequidistant anchored chips are presented in Figure S6b (Supporting Information), showing the newly printed spots intercalated with the previously printed spots. This type of pattern on a single substrate presents an extension of regular arrays as the localization and biochemical information is clustered in certain areas of a single substrate, allowing easier identification and increased/decreased target-probe interactions due to the amount of immobilized targets.

As our goal was to create copies of the patterned chips with the spots following the same distribution, a glass slide was used on the newly fabricated PPL stamps to prevent the contraction after demolding. With this, the protruding stamp features matched the distribution of the anchored chips. The periodic distribution of both anchored chips and polymer pens created repetitive patterns along the $1 \times 1 \mathrm{~cm}^{2}$ sample. If required, alternating patterned chips could be precisely defined by tuning the design of the stamp (with an initial photomask and subsequent microfabrication of the mold), overcoming the possible pattern inconsistencies if using a shrunken PDMS stamp. Having eradicated the contraction, we selectively printed different biomolecule patterns onto functionalized substrates, as presented in Figure 7. Therefore, to test the patterning flexibility of the printing device, Texas Red WGA was patterned creating one-, two-, and five-spot arrays on each anchored chip seen a
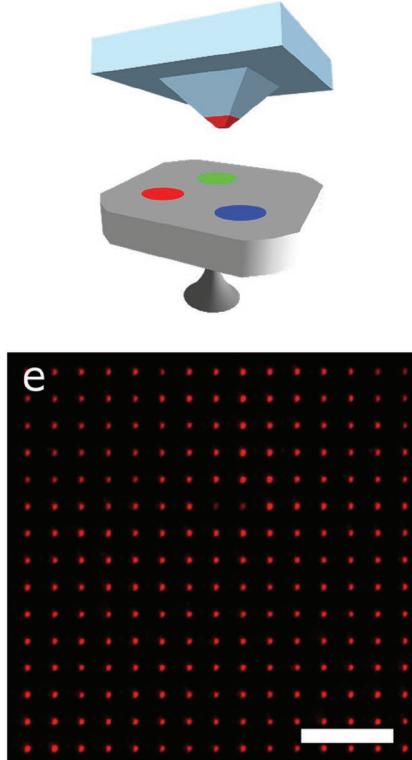
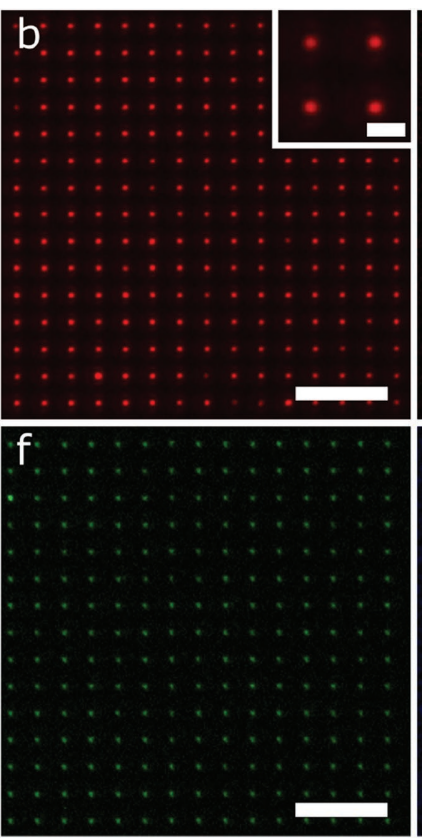
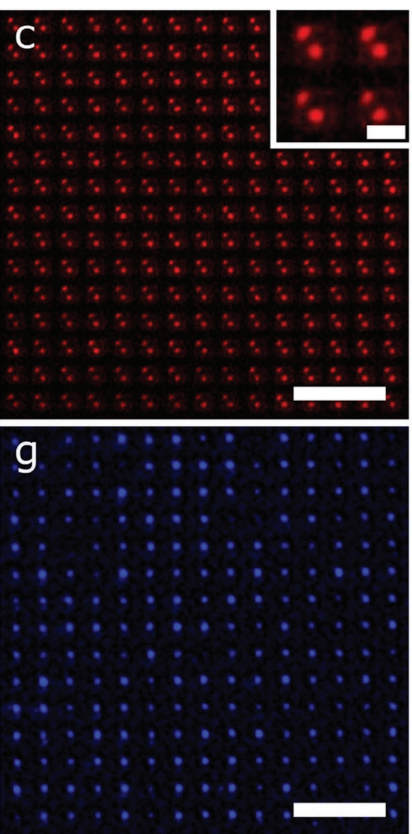
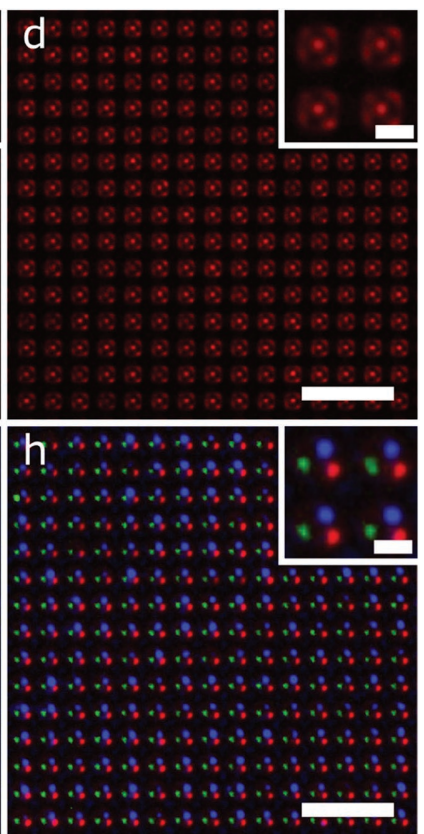

Figure 7. Patterning biomolecules with PPL on the anchored chips. a) The schematic represents the patterning approach. b-d) The fluorescence microscopy images present the consecutive printing processes with a single Texas Red WGA ink yielded individual patterned chips with 1, 2, or up to 5 printed spots, respectively. The versatility of the framework permits the creation of multiplexed arrays on each anchored chip with Texas Red WGA, e) Oregon Green 488 WGA and f) AMCA rabbit anti-goat IgG (H+L), g) spots printed sequentially with PPL. h) The overlay presents the distribution of all the spots along the substrate. Scale bars $=3 \mu \mathrm{m}$ in all insets and $20 \mu \mathrm{m}$ on (b-h) main images. 

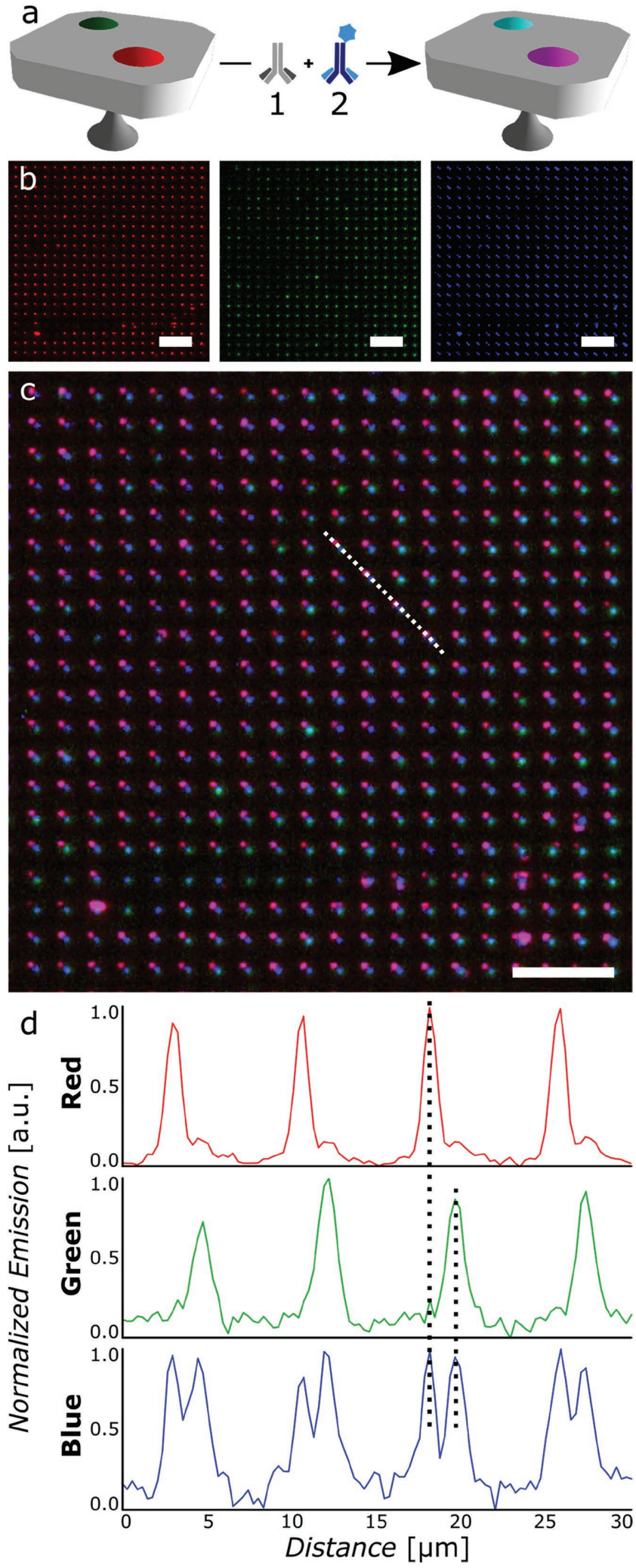

Figure 8. Functional multiplexed biomolecular arrays over the anchored chips. a) Schematic representing the antibody binding assay where goat anti-WGA (1) binds to the WGA spots and is recognized by AMCA rabbit anti-goat $\lg \mathrm{G}(\mathrm{H}+\mathrm{L})(2)$. b) Fluorescence microscopy images of consecutive Texas Red WGA (left) and Oregon Green 488 WGA (middle) spots in Figure $7 \mathrm{~b}-\mathrm{d}$. These multiple patterns on the independent anchored chips were fabricated with the placement of the substrate under the stamp inked with a single initial inking process. All the individual patterns were produced within the $3 \times 3 \mu \mathrm{m}^{2}$ area of each anchored chip, accounting for sub-micrometric displacements between consecutive printing steps and without noticeable ink depletion. To extend the performance of the technique, multiplexed protein patterns were fabricated on top of the anchored chips with the use of two fluorescent-labeled WGAs and a labeled rabbit anti-goat IgG. A PPL stamp was selectively inked in three different areas, each with one of previous inks and subsequently loaded to the printing device. Following the alignment procedure, the inked areas of the stamp were sequentially brought into contact with the substrate. The location of the substrate and stamp was defined to prevent ink overlapping or pattern damage. Figure 7e,f shows the Texas Red WGA and Oregon Green 488 WGA features, respectively, together with Figure $7 \mathrm{~g}$ showing the location of the IgG pattern. The overlay in Figure 7h shows the position of the three biomolecule spots, obtaining a multiplexed pattern on each anchored chip. The footprint of the patterned features in Figure $7 \mathrm{~b}-\mathrm{g}$ was measured using image analysis, obtaining an average edge length of $890 \pm 136 \mathrm{~nm}$. The homogenous distribution was obtained by controlling the printing force to $\approx 1.0-1.5 \mu \mathrm{N}$ per polymer pen throughout the printing procedures, following our previous simulations and spot characterization, described above.

\subsubsection{Functional Protein Array}

The multidimensional protein microarrays can be the foundation for complex biochemical assays. Therefore, to guarantee the integrity of the printed proteins and the integrity of the features, we next developed an antibody binding assay to verify the recognition of the proteins with primary antibodies. The schematic representation in Figure 8a presents the binding assay with the primary goat anti-WGA antibody, which was incubated on the patterned substrate and later incubated with a secondary fluorescently labeled rabbit anti-goat. The initial protein pattern was fabricated with Texas Red and Oregon Green 488 conjugated WGAs. The red, green, and blue fluorescence channels in Figure $8 \mathrm{~b}$ represent the emissions of Texas Red WGA, Oregon Green 488 WGA, and the AMCA rabbit anti-goat, respectively. The merged channels in Figure $8 \mathrm{c}$ show the distribution of the printed spots on top of the anchored microparticles conjugated with the antibodies. To represent the selective recognition of the antibodies, the localized fluorescent emissions were combined between the red, green, and blue channels. Consequently, the red and blue assemble results in a representation in magenta. Similarly, the green and blue emissions are represented in cyan. From the same image, the white dashed line presents the path followed to obtain the emission of the three previous channels, with the normalized emission between

over a same substrate, and the emission of the secondary AMCA rabbit anti-goat IgG $(\mathrm{H}+\mathrm{L})$ antibody (right). c) Overlay image presenting the distribution of the labeled WGA spots with the antibody recognition event. d) Fluorescent emission from the red, green, and blue channels following the dashed line in (c). Scale bars $=20 \mu \mathrm{m}$. 
channels presented in Figure 8d. The matching curve peaks between the red and blue, and green and blue channels are outlined with the connecting dashed line, confirming the correct antibody recognition of each of the patterned spots. This recognition extends the reach of the suspended planar arrays to crucial biological fields where combinatorial bioassays are required such as genomics, ${ }^{[33]}$ proteomics, ${ }^{[34]}$ and drug discovery. ${ }^{[35]}$ The use of microarrays has been the spearhead to work with the vast amount of information required for those crucial biomedical fields. Thus, the creation of replication techniques to produce denser and more complex microarrays will enable faster results with fewer samples.

\section{Conclusions}

In summary, we postulate silicon-based suspended chips as a new kind of highly anisotropic microparticles for the biochemical analysis of small volumes. First, this approach overcomes the designs barrier, opening the door to create chips with the desired physical anisotropies in terms of aspect ratio, faceting, size, and branching, only limited by the minimum feature size achieved by the photolithographic and etching processes. Additionally, our approach permits the inclusion of one or more different physical anisotropies into each individual microparticle. Second, the technology allows extending the functionalization capabilities via wet chemistry, $\mu \mathrm{CP}$, or PPL to precisely print at wafer scale in order to obtain millions of chips with micro- or sub-micrometric molecular patterns with the desired feature size, shape, and distribution. Furthermore, using the PPL technique with the previous reported $100 \mathrm{~nm}$ feature resolution ${ }^{[8]}$ and same pitch, the maximum density could be up to 25 spots $\mu \mathrm{m}^{-2}$, thus greatly miniaturizing the reported multifeature suspended microparticles by two orders of magnitude. ${ }^{[36-38]}$ Future upgrades to the printing setup to print advanced chips (i.e., asymmetric chips) must include sub-100 nm precision $x-y$ piezopositioners in combination with improved interference patterns on the substrates for the nanometric preliminary alignment and subsequent final contact printing. Finally, we believe that the heterogeneity in physical and chemical anisotropies could lead to the fabrication of highly versatile solutions for concrete applications, which could be employed to address fundamental questions in life sciences.

\section{Experimental Section}

Materials and Reagents: $\mathrm{KOH}$, hydrofluoric acid (HF), ethanol, and acetone were purchased from Panreac, Spain. Phosphatebuffered saline (PBS), polyoxyethylenesorbitan monolaurate (Tween 20), sodium phosphate dibasic $\left(\mathrm{Na}_{2} \mathrm{HPO}_{4}\right)$, and 3-glycidoxypropyldimethoxymethylsilane (GOPDMS) were obtained from Sigma-Aldrich, USA. $1 \mathrm{H}, 1 \mathrm{H}, 2 \mathrm{H}, 2 \mathrm{H}$-perfluorooctyltrichlorosilane (PFOTCS) was acquired from Fluka, Switzerland. The Super G Blocking Buffer was purchased from Grace Bio-Labs, USA. Both the AZ 1512 positive photoresist and the AZ $726 \mathrm{MIF}$ developer were obtained from MicroChemicals, Germany, the HIPR 6512 positive photoresist from Fujifilm Electronic Materials, USA, and the Sioetch MT 06/01VLSI solution from Selectipur, Switzerland. The 300 and $525 \mu \mathrm{m}$ thick P-type $\langle 100\rangle$ silicon wafers were ordered from Okmetric, Finland, and the $300 \mu \mathrm{m}$ thick P-type $\langle 100\rangle$ silicon wafer with a $1 \mu \mathrm{m}$ thick $\mathrm{SiO}_{2}$ layer from NTB, Switzerland.
PDMS (Sylgard 184) was purchased from Dow Corning, USA. Both WGA labeled with either Texas Red or Oregon Green 488 were purchased from Invitrogen, USA, and the goat anti-WGA and AMCA (7-amino-4methylcoumarin-3-acetic acid) rabbit anti-goat IgG $(\mathrm{H}+\mathrm{L})$ from Vector Laboratories, USA. The 5OG-3 strand was labeled with Oregon Green 488 in the 5' end, had the sequence: 5'-CAA GAC CGA GCT GAT CAA ACC-3', and was acquired from Metabion, Germany. The FP- $\mathrm{NH}_{2}-\mathrm{T}$ was modified with $\mathrm{NH}_{2}-\mathrm{C}_{6}$ in the $5^{\prime}$ end and with tetramethylrhodamine (TAMRA) in the $3^{\prime}$ end, had the sequence $5^{\prime}-10$ (T) GGT TTC ATC AGC TCC GTC TTG-3', and was purchased from biomers.net, Germany. All reagents were used as received and without further purification.

Fabrication of Anchored Chips: A $500 \mu \mathrm{m}$ thick P-type $\langle 100\rangle$ silicon wafer was wet-oxidized at $1100{ }^{\circ} \mathrm{C}$ to grow a $1 \mu \mathrm{m}$ thick $\mathrm{SiO}_{2}$ layer. Subsequently, a $1.2 \mu \mathrm{m}$ thick layer of the HIPR 6512 photoresist was spun onto the oxidized wafer and exposed to UV light using a MA56 mask aligner (Karl Süss, Germany) through a photomask to define the lateral dimensions and spatial distribution of the chips. After developing and baking the resist at $200{ }^{\circ} \mathrm{C}$ for $30 \mathrm{~min}$, the exposed $\mathrm{SiO}_{2}$ layer was dry-etched with a $\mathrm{C}_{2} \mathrm{~F}_{6} / \mathrm{CHF}_{3}$ mixture in a Drytek Quad 484 RIE (Drytek, USA). Finally, the anchors under the chips were defined using quasiisotropic etching in a $\mathrm{SF}_{6} / \mathrm{C}_{4} \mathrm{~F}_{8}$ atmosphere in an A-601E DRIE (Alcatel, France), and the remaining photoresist stripped in a TePla 300-E plasma system (Technics Plasma, USA).

Surface Activation of the Anchored Chips: An epoxy-terminated SAM was deposited over the top or all the surfaces of the $\mathrm{SiO}_{2}$ anchored chips via contact printing or wet chemistry, respectively. First, the substrate was plasma-activated for $30 \mathrm{~s}$ at $30 \mathrm{~W}$ in a plasma cleaner (Harrick Plasma, USA) and subsequently printed for $5 \mathrm{~min}$ with a flat PDMS stamp inked with a $2 \%$ GOPDMS ethanolic solution or incubated with the same solution for $20 \mathrm{~min}$. The substrate was later rinsed with absolute ethanol and cured at $75{ }^{\circ} \mathrm{C}$ for $1 \mathrm{~h}$, afterward rinsed with absolute ethanol and blown dry with $\mathrm{N}_{2}$.

Surface Modification with Wet Chemistry: The epoxy-activated substrate was incubated with a protein suspended in a $30 \times 10^{-3} \mathrm{M} \mathrm{Na} \mathrm{NPO}_{4}$ aqueous solution at a final concentration of $40 \mu \mathrm{g} \mathrm{mL}$. The substrate was left to react in the dark for $1 \mathrm{~h}$. Later, the substrate was rinsed with a PBS $0.1 \%$ Tween 20 (PBS-T) solution and finally Milli-Q water.

Fabrication of the Molds and PDMS Stamps: To fabricate the PPL mold, the same procedure to create the anchored chips was followed using a $525 \mu \mathrm{m}$ thick P-type $\langle 100\rangle$ silicon wafer until the etching process. The same photomask was used to guarantee one inverted pyramid per anchored chip. To create the inverted pyramids, the exposed silicon was anisotropically etched by submerging the wafer in a $40 \% \mathrm{KOH}$ solution at $75{ }^{\circ} \mathrm{C}$ for $4 \mathrm{~min}$. The remaining $\mathrm{SiO}_{2}$ layer was later stripped with the commercial Sioetch remover. Alternatively, to fabricate the mold with lines, the $300 \mu \mathrm{m}$ thick P-type $\langle 100\rangle$ silicon wafer with a $1 \mu \mathrm{m}$ thick $\mathrm{SiO}_{2}$ layer was coated with $1.4 \mu \mathrm{m}$ of $\mathrm{AZ}$ photoresist and backed at $95{ }^{\circ} \mathrm{C}$ for $2 \mathrm{~min}$. The coated wafer was introduced into a DWL 66FS maskless lithography equipment (Heidelberg Instruments Mikrotechnik, Germany) and the features were defined by the selective exposition to a $405 \mathrm{~nm}$ laser. The exposed photoresist was later developed with the $\mathrm{AZ}$ developer. Finally, the exposed $\mathrm{SiO}_{2}$ was wet-etched in a $\mathrm{HF}$ solution, and the remaining photoresist eliminated with acetone. To avoid the PDMS from sticking to the molds, both PPL and line molds were plasma activated for $30 \mathrm{~s}$ at $30 \mathrm{~W}$ and exposed to a saturated PFOTCS atmosphere for $1 \mathrm{~h}$. To fabricate the PDMS stamps a 10:1 w/w prepolymer-catalyst mixture was degassed and poured over the silanized molds. To prevent the contraction of the PDMS after peeling from the mold, plasma-activated glass slides (Corning, USA) were placed over the uncured PDMS and left to cross-link at $75{ }^{\circ} \mathrm{C}$ for $1 \mathrm{~h}$ obtaining a stamp with a thickness of $\approx 100 \mu \mathrm{m}$.

Numerical Simulation: The mechanical deformation of the polymer pen was simulated using the FEM with ANSYS Multiphysics (Release 14.0, www.ansys.com). The polymer pen and contact substrate were modeled using the 3D SOLID185 element. To limit the deformation of the contact substrate, a Young's modulus of $169 \mathrm{GPa}$ was used, along the range of $0.34-0.75 \mathrm{MPa}$ for the PDMS pen. A contact pair was defined between the tip of the pen and the substrate, and varying vertical 
loads were applied over the pen. The change in the contact area with varying loads was simulated.

Alignment between Stamp and Substrate: A custom-made PPL printing device was used as described previously. ${ }^{[19]}$ Shortly, the substrate was immobilized on a movable stage with sub-micrometer resolution on both $x$ and $y$ axes. The stamp was fixed on a specialized holder and was brought into contact with the substrate using a piezoelectric actuator. The roll, pitch, and yaw movements were controlled with independent actuators to guarantee a parallel patterning process. The patterning process was monitored with both, a dedicated optical monitoring and a pressure sensing system, to track in real time the movement of the stamp and printing pressure, respectively.

Printing Multiplexed Biomolecular Patterns: The silanized substrates were vacuum-fixed to the substrate holder in the PPL printing device. The PDMS stamps were fixed to the stamp holder with vacuum and positioned above the substrate. The PDMS stamp was divided into one, two, or three patterning areas which were inked with a single biomolecular ink for $15 \mathrm{~min}$. The Texas Red WGA and Oregon Green 488 WGA inks were used at a concentration of $40 \mu \mathrm{g} \mathrm{mL}^{-1}$, the AMCA rabbit anti-Goat IgG $(\mathrm{H}+\mathrm{L})$ was dissolved to a final concentration of $15 \mu \mathrm{g} \mathrm{mL} \mathrm{L}^{-1}$, and the DNA strand was used at a $5 \times 10^{-6} \mathrm{M}$ concentration. All inks were independently suspended in a $30 \times 10^{-3} \mathrm{M} \mathrm{Na}_{2} \mathrm{HPO}_{4}$ aqueous solution. After inking, the stamps were rinsed with Milli-Q water (Millipore, USA) and placed in contact on the desired area of the substrate for a standardized time of $2 \mathrm{~min}$. For multiplexed patterns, the substrate was sequentially printed, translating it under the independently inked area of the PDMS stamp. The patterned substrate was left to react in a dark, humid chamber at room temperature for $1 \mathrm{~h}$ before blocking the exposed epoxy moieties with the Super G Blocking Buffer. To finalize the patterning process, the substrate was rinsed with Milli-Q water and blown dry with $\mathrm{N}_{2}$.

Antibody Binding Assay: A drop of a $10 \times 10^{-6} \mathrm{M}$ solution of primary goat anti-WGA in sterile PBS was placed over the patterned substrate and incubated in a dark chamber for $1 \mathrm{~h}$. Subsequently, unbound antibody was washed initially with a PBS-T and afterward with PBS. The substrate was later incubated with a $1 \times 10^{-6} \mathrm{M}$ AMCA rabbit anti-Goat IgC $(\mathrm{H}+\mathrm{L})$ antibody solution in PBS and left to react for $1 \mathrm{~h}$. Finally, the substrate was rinsed with a PBS-T and Milli-Q water.

Image Acquisition and Processing: Scanning electron microscopy (SEM) characterization was done in a Nova NanoSEM 230 microscope (FEI Co., USA). The atomic force microscopy (AFM) topographical images were obtained using a Dimension 3100 microscope (Veeco Instruments, USA) in tapping mode using a rectangular NSC15/AIBS silicon tip with $k=40 \mathrm{~N} \mathrm{~m}^{-1}$ from MikroMasch (Wetzlar, Germany). AFM images were processed with the WSxM software. ${ }^{[39]}$ All fluorescence emissions were acquired using an Eclipse E100 fluorescence microscope (Nikon, Japan) and the images were processed using the Image) software (NIH, USA).

\section{Supporting Information}

Supporting Information is available from the Wiley Online Library or from the author.

\section{Acknowledgements}

This work was supported by the EU ERDF (FEDER) funds and the Spanish Government Grant Nos. TEC2014-51940 and TEC2015-70104-P. The Nanobioengineering SIC-BIO group was supported with the grant 2014 SGR 1442 from the Generalitat de Catalunya. The CIBER-BBN was financed by the Instituto de Salud Carlos III with assistance from the EU ERDF. The authors also thank the cleanroom staff of IMB-CNM for fabrication of the chips.

Received: November 10, 2016 Revised: December 21, 2016 Published online:
[1] S. F. Kingsmore, Nat. Rev. Drug Discovery 2006, 5, 310

[2] G. Arrabito, B. Pignataro, Anal. Chem. 2012, 84, 5450.

[3] A. Q. Emili, G. Cagney, Nat. Biotechnol. 2000, 18, 393.

[4] D. Weinrich, P. Jonkheijm, C. M. Niemeyer, H. Waldmann, Angew. Chem. Int. Ed. 2009, 48, 7744

[5] V. Romanov, S. N. Davidoff, A. R. Miles, D. W. Grainger, B. K. Gale, B. D. Brooks, Analyst 2014, 139, 1303.

[6] F. Brinkmann, M. Hirtz, A. M. Greiner, M. Weschenfelder B. Waterkotte, M. Bastmeyer, H. Fuchs, Small 2013, 9, 3266.

[7] D. Qin, Y. Xia, G. M. Whitesides, Nat. Protoc. 2010, 5, 491.

[8] F. Huo, Z. Zheng, G. Zheng, L. R. Giam, H. Zhang, C. A. Mirkin, Science 2008, 321, 1658.

[9] R. D. Piner, J. Zhu, F. Xu, S. Hong, C. A. Mirkin, Science 1999, 283, 661.

[10] X. Liao, A. B. Braunschweig, Z. Zheng, C. A. Mirkin, Small 2010, 6, 1082.

[11] D. J. Eichelsdoerfer, K. A. Brown, M. X. Wang, C. A. Mirkin, J. Phys. Chem. B 2013, 117, 16363.

[12] Z. Zheng, W. L. Daniel, L. R. Giam, F. Huo, A. J. Senesi, G. Zheng C. A. Mirkin, Angew. Chem. 2009, 48, 7626.

[13] R. Kumar, S. Weigel, R. Meyer, C. M. Niemeyer, H. Fuchs, M. Hirtz Chem. Commun. 2016, 52, 12310.

[14] K. Braeckmans, S. C. De Smedt, Nat. Mater. 2010, 9, 697.

[15] S. Rahmani, S. Saha, H. Durmaz, A. Donini, A. C. Misra, J. Yoon, J. Lahann, Angew. Chem. 2014, 126, 2364.

[16] A. Bruckbauer, D. Zhou, D. J. Kang, Y. E. Korchev, C. Abell, D. Klenerman, J. Am. Chem. Soc. 2004, 126, 6508.

[17] I. Tsarfati-BarAd, U. Sauer, C. Preininger, L. A. Gheber, Biosens. Bioelectron. 2011, 26, 3774.

[18] K. L. Christman, V. D. Enriquez-Rios, H. D. Maynard, Soft Matter 2006, 2, 928.

[19] N. Torras, J. P. Agusil, P. Vázquez, M. Duch, A. M. Hernández-Pinto, J. Samitier, E. J. de la Rosa, J. Esteve, T. Suárez, L. Pérez-García, J. A. Plaza, Adv. Mater. 2016, 28, 1449.

[20] L. R. Giam, M. D. Massich, L. Hao, L. S. Wong, C. C. Mader C. A. Mirkin, Proc. Natl. Acad. Sci. USA 2012, 109, 4377.

[21] S. C. Glotzer, M. J. Solomon, Nat. Mater. 2007, 6, 557.

[22] U. Bog, F. Brinkmann, H. Kalt, C. Koos, T. Mappes, M. Hirtz, H. Fuchs, S. Köber, Small 2014, 10, 3863.

[23] C. Thibault, V. Le Berre, S. Casimirius, E. Trévisiol, J. François, C. Vieu, J. Nanobiotechnol. 2005, 3, 7.

[24] D. J. Eichelsdoerfer, X. Liao, M. D. Cabezas, W. Morris, B. Radha, K. A. Brown, L. R. Giam, A. B. Braunschweig, C. A. Mirkin, Nat. Protoc. 2013, 8, 2548

[25] X. Liao, A. B. Braunschweig, C. A. Mirkin, Nano Lett. 2010, 10, 1335

[26] Z. Xie, Y. Shen, X. Zhou, Y. Yang, Q. Tang, Q. Miao, J. Su, H. Wu, Z. Zheng, Small 2012, 8, 2664.

[27] B. R. Takulapalli, M. E. Morrison, J. Gu, P. Zhang, Nanotechnology 2011, 22, 285302

[28] J. M. Hong, F. M. Ozkeskin, J. Zou, J. Micromech. Microeng. 2007 $18,15003$.

[29] H. Noh, G.-E. Jung, S. Kim, S.-H. Yun, A. Jo, S.-J. Kahng, N.-J. Cho, S.-J. Cho, Small 2015, 11, 4526.

[30] D. Armani, C. Liu, N. Aluru, IEEE Int. Conf. Micro Electro Mech. Syst., 12th 1999, 222.

[31] C. Moraes, Y. Sun, C. A. Simmons, J. Micromech. Microeng. 2009, 19,65015

[32] S. W. Lee, S. S. Lee, Microsyst. Technol. 2007, 14, 205.

[33] V. Trevino, F. Falciani, H. Barrera-Saldaña, Mol. Med. 2007, 13, 527.

[34] H. Sun, G. Y. J. Chen, S. Q. Yao, Chem. Biol. 2013, 20, 685.

[35] H. Ma, K. Y. Horiuchi, Drug Discovery Today 2006, 11, 661.

[36] K. Maeda, H. Onoe, M. Takinoue, S. Takeuchi, Adv. Mater. 2012, 24, 1340

[37] J. Lee, P. W. Bisso, R. L. Srinivas, J. J. Kim, A. J. Swiston, P. S. Doyle, Nat. Mater. 2014, 13, 524

[38] H. Lee, J. Kim, H. Kim, J. Kim, S. Kwon, Nat. Mater. 2010, 9, 745.

[39] I. Horcas, R. Fernández, J. M. Gómez-Rodríguez, J. Colchero, J. Gómez-Herrero, A. M. Baro, Rev. Sci. Instrum. 2007, 78, 13705. 